\title{
A Combined Statistical and Biomechanical Model for Estimation of Intra-operative Prostate Deformation
}

\author{
Ashraf Mohamed ${ }^{1,2}$, Christos Davatzikos ${ }^{1,2}$, and Russell Taylor ${ }^{1}$ \\ 1 CISST NSF Engineering Research Center \\ Department of Computer Science, Johns Hopkins University \\ rht@cs.jhu.edu \\ http://cisstweb.cs.jhu.edu/ \\ ${ }^{2}$ Center for Biomedical Image Computing, Department of Radiology \\ Johns Hopkins University School of Medicine \\ \{ashraf, hristos\}@rad.jhu.edu \\ http://cbic.jhoc1.jhmi.edu/
}

\begin{abstract}
An approach for estimating the deformation of the prostate caused by transrectal ultrasound (TRUS) probe insertion is presented. This work is particularly useful during brachytherapy procedures, in which planning for radioactive seed insertion is performed on preoperative scans, and significant deformation of the prostate can occur during the procedure. The approach makes use of a patient specific biomechanical model to run simulations for TRUS probe insertion, extract the main modes of the deformation of the prostate, and use this information to establish a deformable registration between 2 orthogonal cross-sectional ultrasound images and the preoperative prostate. In the work presented here, the approach is tested on an anatomy-realistic biomechanical phantom for the prostate and results are reported for 5 test simulations. More than $73 \%$ of maximum deformation of the prostate was recovered, with the estimation error mostly attributed to the relatively small number of biomechanical simulations used for training.
\end{abstract}

\section{Introduction}

Transrectal ultrasound (TRUS) guided brachytherapy is one of the common therapy alternatives for prostate cancer. The goal of the procedure is to insert a number of radioactive seeds at specific locations into the prostate tissue by using surgical needles. The locations and number of seeds within the prostate gland are decided by means of a surgical planning software that makes use of preoperative volumetric scans of the prostate, typically CT or MRI.

During brachytherapy, several factors can cause deformation of the prostate gland from its preoperative shape. These factors include insertion of the ultrasound probe inside the rectum, insertion of the surgical needles, edema, and change in the patient's posture between the preoperative and the intraoperative conditions [1]. This deformation of the prostate from the preoperative condition induces uncertainties in the radioactive seeds insertion locations, which are the result of planning on preoperative images. Thus, deformation of the prostate can 
affect the dose distribution in and around the prostate and therefore adversely affect the outcome of the procedure [2].

The goal of the work reported here is to describe a framework for estimating the deformation of the prostate based on sparse data from 2D ultrasound images that can be obtained during a typical brachytherapy procedure. Such estimator can update the preoperative plan to account for deformations thereby reducing the uncertainty in the radioactive seed insertion locations. Our approach builds upon [3], in which a general framework for statistical estimation of intra-operative deformations was presented. The problem of estimation of the deformed shape of the prostate at each probe location can therefore be cast as a deformable $2 \mathrm{D} / 3 \mathrm{D}$ registration problem [4, i.e. registration of a $3 \mathrm{D}$ preoperative image to two cross-sections of the deformed volume of the same patient.

To obtain the main modes of deformation of the prostate under TRUS probe insertion, a patient-specific biomechanical model is constructed from segmented preoperative images. The main modes of deformation are extracted by performing Principal Component Analysis (PCA) on a small number of deformed shapes resulting from simulations for TRUS probe insertion which are run on the biomechanical model. Each of the simulations corresponds to specific insertion angles and insertion depth of the TRUS probe. In order to further simplify the estimator, we derive an analytic representation of the principal modes of deformation (coefficients of the principal modes) as a function of the probe insertion angles and insertion depth. Our goal is to develop a fast, statistically based model, which can be used in real-time to track deformations. This model is trained on computationally intensive biomechanical simulations, which are performed preoperatively.

In Section 2, the construction of the prostate phantom, the deformable prostate model, and the estimator are described. In the preliminary work of this paper, the proposed approach is tested on 5 biomechanical simulations that are not used for training. Simulated ultrasound prostate contours are obtained from the deformed prostate and are used by the estimator to estimate those deformed shapes. The results reported in Section 3 indicate good accuracy in the estimation of deformed shapes. In Section 4, we discuss how the current work can be extended to deal with real patient data and to deal with different subjects.

\section{Methods}

In this section, the construction of the estimator of the deformed prostate shapes is detailed. First, the biomechanical model used for simulation of prostate deformation is described. This biomechanical model is patient specific and is constructed from the patient's segmented preoperative CT or MRI scan. In the work presented here, a biomechanical model of an anatomy-realistic prostate phantom is used instead. This provides a means for validating the estimates of the deformed prostate by comparing them to the true deformed shapes of the prostate, which are not usually available for real patients unless intra-operative imaging is used. We use the patient's specific biomechanical model to run a 
number of biomechanical simulations with different insertion depths and entry angles of the TRUS probe, thereby constructing a number of deformed shapes of the same prostate. The simulations involved the entry angles of the probe to account for misalignment between the axes of the rectum and the probe.

From the simulated deformed shapes, we extract the principal modes of deformation for that prostate under TRUS probe insertion. Noting the dependency between the modes of deformation and the parameters of the simulations (the insertion depth and angles of the TRUS probe) a functional approximation was sought by fitting a $3^{\text {rd }}$ degree Bernstein polynomial. Therefore any deformed prostate shape can be described in terms of the principal modes of deformation and their corresponding principal components which are in turn directly related to the insertion angles and insertion depth of the probe. From transaxial and sagittal sections of the prostate obtained through the TRUS probe deformable $2 \mathrm{D} / 3 \mathrm{D}$ registration is established between the estimated deformed prostate shapes and the images obtained intra-operatively.

\subsection{Biomechanical Model}

A patient specific biomechanical model is needed for estimation of the range of deformations of the prostate. Finite element biomechanical meshes can be automatically generated from segmented images of the patient (e.g. [5]6]). For the model to be able to capture the deformation of the prostate accurately, it should include structures such as the prostate, surrounding tissues, the rectum, and the surrounding bony structures (sacrum and pubic arch) that control the boundary conditions. In this preliminary work reported here, we used an anatomy-realistic 3D phantom of the prostate and the surrounding structures for reasons stated earlier. A side view and a 3D rendered view of the phantom are shown in Figure 1.

The phantom is composed mainly of a block of soft tissue of dimensions $12 \mathrm{~cm} \times 16 \mathrm{~cm} \times 12 \mathrm{~cm}$. The prostate is modeled as an egg shaped structure of dimensions $3 \mathrm{~cm} \times 3 \mathrm{~cm} \times 3.5 \mathrm{~cm}$. The rectum is modeled as a straight cylinder with circular cross section of radius $0.5 \mathrm{~cm}$ that runs $0.25 \mathrm{~cm}$ below the lower surface of the prostate. The surfaces of the sacrum, pubic arch, were generated using a spline curve extruded in $3 \mathrm{D}$. The sacrum and the pubic arch are assumed to be pinned and therefore they define the boundary conditions for the problem. No other boundary conditions are imposed on any other structure. A tetrahedral mesh was automatically generated for the prostate and surrounding soft tissue within Abaqus CAE Finite Element (FE) environment [7] which is also used for solving the biomechanical FE model.

Even though there is evidence that most soft tissues exhibit non-linear material behavior, a linear material model was used in many studies dealing with biomechanical behavior of soft tissue (e.g. [5]). A linear material model is typically chosen because it produces faster results compared to a non-linear material model and it is easier to implement. The values of material parameters, (e.g. Young's modulus and Poisson's ratio for a linear material model) vary widely from one tissue type to another and from one person to another for the same soft tissue type, especially with the presence of tissue anomaly such as cancer. To our 


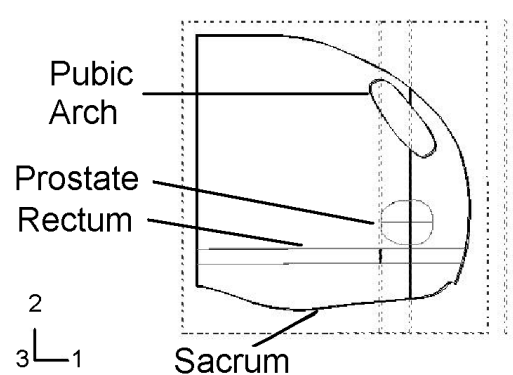

(a)

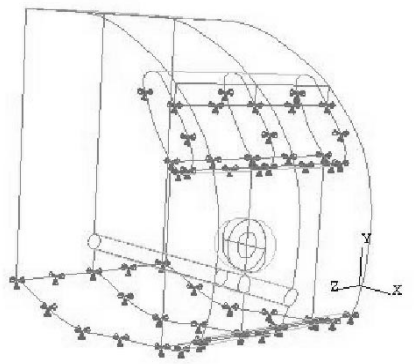

(b)

Fig. 1. The biomechanical prostate phantom. (a) $2 \mathrm{D}$ profile. (b) $3 \mathrm{D}$ wireframe with no-displacement boundary conditions imposed on the sacrum and the pubic arch.

knowledge the material parameter values for the prostate have not been determined experimentally or otherwise for the in-vivo human prostate. In [1] a linear material model was used for the prostate with different stiffness values for the central gland and the peripheral zone. A linear material model is only valid for small deformations and therefore offers limited accuracy in problems that involve large deformation. In the work presented here, since the expected deformation is large, we used a homogeneous Mooney-Rivlin non-linear material model for the prostate tissue with an initial Young's Modulus (stiffness) value $E=2 \mathrm{kPa}$ and an almost incompressible behavior. This is consistent with the values used in [1] for the peripheral zone of the prostate. For the soft tissue surrounding the prostate, a Mooney-Rivlin material model was also assumed but with an initial stiffness that is 10 times as large as that assumed for the prostate tissue. Such values of material parameters produced deformations that are consistent with observed deformation in real TRUS images. Recently, Magnetic Resonance Elastography has been proposed for in-vivo estimation of material parameter values [8]. If accurate patient specific material parameter values are known, they can be used directly in the model. In section 4 we discuss how our approach can be extended to deal with deformations even if the material parameters were not known accurately, but are known to lie in a certain range. It is important to note that we do not need exact knowledge of the elastic parameters since our goal is to develop a statistical prior model that will follow the actual deformation in TRUS images rather than totally predict the deformation of the prostate.

During TRUS-guided prostate brachytherapy, the ultrasound probe is inserted at increasing depths with known constant displacements in between. This causes the dilation of the rectum and exertion of pressure on the surrounding tissues, including the prostate. The displacement of the probe along its axis measured from the start of the rectum as a reference point is denoted by the variable $u$. The angles $\phi_{2}$ and $\phi_{3}$ denote the rotation angles around the $2^{\text {nd }}$ and $3^{r d}$ coordinate axes respectively (see Figure 1) and are referred to here as the entry angles of the probe. 


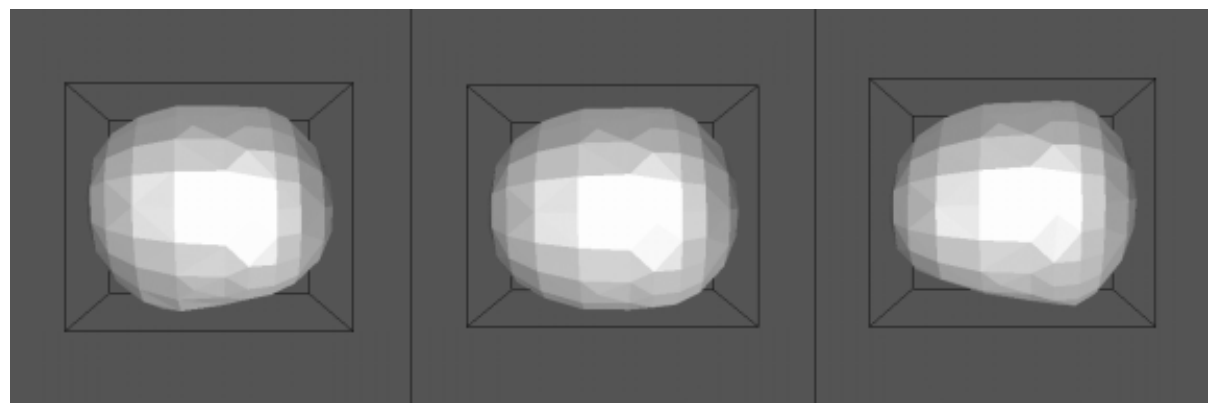

Fig. 2. The mean shape of the statistical model is shown in the middle, with added -3 standard deviations (left) and +3 standard deviations (right) of the $2^{\text {nd }}$ mode of the deformation.

\subsection{Prostate Deformable Model}

For training purpose of the deformable statistical model of the prostate, simulations of TRUS probe insertion with different entry angles spanning the range -4 to 4 degrees were performed. A total of 25 such simulations were performed, each with 9 corresponding probe displacements that simulate imaging of the whole prostate in 2D cross sections. Displacements of the probe were $0.5 \mathrm{~cm}$ in between, which is consistent with staging in available TRUS systems used for brachytherapy. A total of 225 deformed prostate shapes were therefore available from these simulations. For each simulation, coordinates of the finite element node locations of each of the deformed shapes were assembled into a vector $\mathbf{q}$ that represents the deformed shape.

Principal Component Analysis (PCA) 9] was performed on the deformed prostate shapes to obtain the main modes of deformation of the prostate. Therefore, any of the simulated deformed shapes can be approximated by

$$
\mathbf{q}=\mu+\sum_{i=1}^{M} \alpha_{i} \mathbf{x}_{i}
$$

where $\mu$ is the mean shape of the deformed prostate, $\mathbf{x}_{i}$ are the principal modes of deformation, $\alpha_{i}$ are the expansion coefficients, and $M$ is the number of retained modes of deformation. More than $99 \%$ of the variation in the training samples was explained by only the first 6 modes of the deformation, and therefore $M=6$ was used for the results reported in this work.

Some of the modes of deformation were highly correlated with the physical parameters of the biomechanical simulations (modes 1, 2 and 4). In Figure 2, the second mode of deformation of the prostate is shown. It is clear from the figure that the second principal component correlates well (correlation coefficient of 0.88 ) with the displacement of the TRUS, $u$. Similarly, modes 1 and 4 correlated highly (correlation coefficients $\geq 0.67$ ) with $\phi_{2}$ and $\phi_{3}$ respectively. Given this observation, a functional relationship was assumed between the principal components of the deformation and the biomechanical simulation parameters, i.e. 


$$
\alpha_{i}=f_{i}\left(u, \phi_{2}, \phi_{3}\right), \quad 1 \leq i \leq M .
$$

Linear least squares fitting was used to approximate each $f_{i}$ by fitting $3^{\text {rd }}$ degree Bernstein polynomials [10] for each of the principal components in terms of the simulation parameters. Therefore a deformed shape is related to the biomechanical simulation parameters by

$$
\begin{aligned}
\mathbf{q} & =G\left(u, \phi_{2}, \phi_{3}\right) \\
& =\mu+\sum_{i=1}^{M} f_{i}\left(u, \phi_{2}, \phi_{3}\right) \mathbf{x}_{i}
\end{aligned}
$$

To evaluate the error introduced by the fitting of Bernstein polynomials for the functions $f_{i}$ of equation (3), true deformed shapes that resulted from biomechanical simulations were compared to the deformed shapes constructed by equation (3) for the same simulation parameter values. The maximum error was $0.09 \mathrm{~cm}$, while the maximum deformation encountered in the simulated shapes was $0.7 \mathrm{~cm}$. Therefore, a maximum error of $12.9 \%$ was introduced in the training samples by the approximation of equation (3) and using a finite number of deformation modes $(M=6)$.

\subsection{Estimation of Deformed Shapes}

During prostate brachytherapy, and before inserting any radioactive seeds, a number of $2 \mathrm{D}$ ultrasound images are usually obtained to cover the whole prostate. The displacements between the locations at which the images are obtained are known since a mechanical stepper is typically used to advance the TRUS probe. The goal is to estimate the deformed shape of the prostate at each of those probe locations. If the known displacement between consecutive probe locations is denoted by $\triangle u$, then the deformed shapes are given by

$$
\mathbf{q}_{j}=G\left(u_{o}+\Delta u(j-1), \phi_{2_{o}}, \phi_{3_{o}}\right) \quad 1 \leq j \leq K .
$$

where $K$ is the number of probe locations, $u_{o}$ the displacement for the first probe location, $\phi_{2_{o}}$ and $\phi_{3_{o}}$ are the insertion angles for the probe. Thus, if $u_{o}, \phi_{2_{o}}$ and $\phi_{3 o}$ were known then the whole set of deformed shapes at different locations of the probe will be available.

In the work presented here, it is assumed that at each location of the probe, 2 orthogonal images of the prostate are available. These images are readily obtained by most TRUS probes currently in use for brachytherapy. From each image, coordinates of points on the surface of the deformed prostate can be extracted using manual or automatic outlining. Let the $3 \mathrm{D}$ coordinates of the points obtained at the $j^{t h}$ location of the probe relative to the ultrasound crystal be denoted by $V_{j}$, where $1 \leq j \leq K$. A coordinate frame transformation relates the coordinate frame of the crystal to the coordinate frame in which the simulations were performed. This transformation can be computed in terms of the geometry of the probe, the parameters $u, \phi_{2}$, and $\phi_{3}$, and $\mathbf{t}_{o}$, an unknown translation between the coordinate frames. Let this frame transformation for the $j^{\text {th }}$ location 
of the probe be denoted by $\mathcal{T}_{j}$. Given an estimate of $\mathcal{T}_{j}$, let $V^{t}{ }_{j}$ denote the points $V_{j}$ transformed into the simulation coordinate frame by $\mathcal{T}_{j}$. Also, let the sum of squared distances between the points $V^{t}{ }_{j}$ and their closest corresponding points on the deformed surface $\mathbf{q}_{j}$ be denoted by $\mathcal{E}_{j}\left(u_{o}, \phi_{2_{o}}, \phi_{3_{o}}, \mathbf{t}_{o}\right)$. Therefore, we seek the values $\hat{u}_{o}, \hat{\phi}_{2_{o}}, \hat{\phi}_{3 o}$, and $\hat{\mathbf{t}}_{o}$ that minimize the sum of square errors:

$$
\left(\hat{u}_{o}, \hat{\phi}_{2 o}, \hat{\phi}_{3 o}, \hat{\mathbf{t}}_{o}\right)=\arg \min \sum_{j=1}^{K} \mathcal{E}_{j}\left(u_{o}, \phi_{2 o}, \phi_{3 o}\right)
$$

Using $\hat{u}_{o}, \hat{\phi}_{2_{o}}, \hat{\phi}_{3}$ in equation 4 yields the estimates of the deformed shapes, $\hat{\mathbf{q}}_{j}, 1 \leq j \leq K$. The optimization problem is solved using the Nelder-Mead nonlinear optimization method [1] from within the Matlab environment. Similar to the approach in 4], the optimization for the parameters $u_{o}, \phi_{2_{o}}, \phi_{3_{o}}$, and $\mathbf{t}_{o}$ is performed at 2 different alternating steps for deformable $2 \mathrm{D} / 3 \mathrm{D}$ registration and pure translation.

\section{Results}

Five simulations of TRUS probe insertion were performed at parameter values $u_{o}, \phi_{2_{o}}$, and $\phi_{3_{o}}$ that were different from those used for the training but within the range of training values. A pair of orthogonal simulated TRUS prostate image contours were generated at each location of the probe. The estimator described above was then used to obtain the deformed shapes of the prostate at each location of the probe.

We computed the estimation error defined as the difference between the estimated deformed prostate shape and true deformed shape obtained by biomechanical simulation:

$$
\hat{\mathbf{e}}_{j}=\hat{\mathbf{q}}_{j}-\mathbf{q}_{j} \quad 1 \leq j \leq K
$$

We also computed the reconstruction error for the deformed shapes, defined as the difference between a deformed shape and its best possible reconstruction in the space spanned by the retained principal modes of the deformation:

$$
\check{\mathbf{e}}_{j}=\check{\mathbf{q}}_{j}-\mathbf{q}_{j} \quad 1 \leq j \leq K
$$

where $\check{\mathbf{q}}_{j}=\mu+\sum_{i=1}^{M} \check{\alpha}_{i} \mathbf{x}_{i}$ and $\check{\alpha}_{i}$ are obtained by projecting the deformed shape $q_{j}$ on the orthogonal principal modes $\mathbf{x}_{i}$. The estimation error can therefore be decomposed into 2 orthogonal components [12]

$$
\hat{\mathbf{e}}_{j}=\check{\mathbf{e}}_{j}+\tilde{\mathbf{e}}_{j}
$$

The reconstruction error $\check{\mathbf{e}}_{j}$, is due to the inability of representing the deformed shape $\mathbf{q}_{j}$ as the sum of the mean and a linear combination of the principal modes of deformation, while the error $\tilde{\mathbf{e}}_{j}$ is due to inability of estimating the deformed shape perfectly from the 2D information provided by the TRUS images, and due the approximation of equation (31). The maximum estimation error and reconstruction error for each of the simulations are shown in Figure 3. In the worst test case (case number 4 ), the max estimation error was $26.7 \%$ of the maximum 


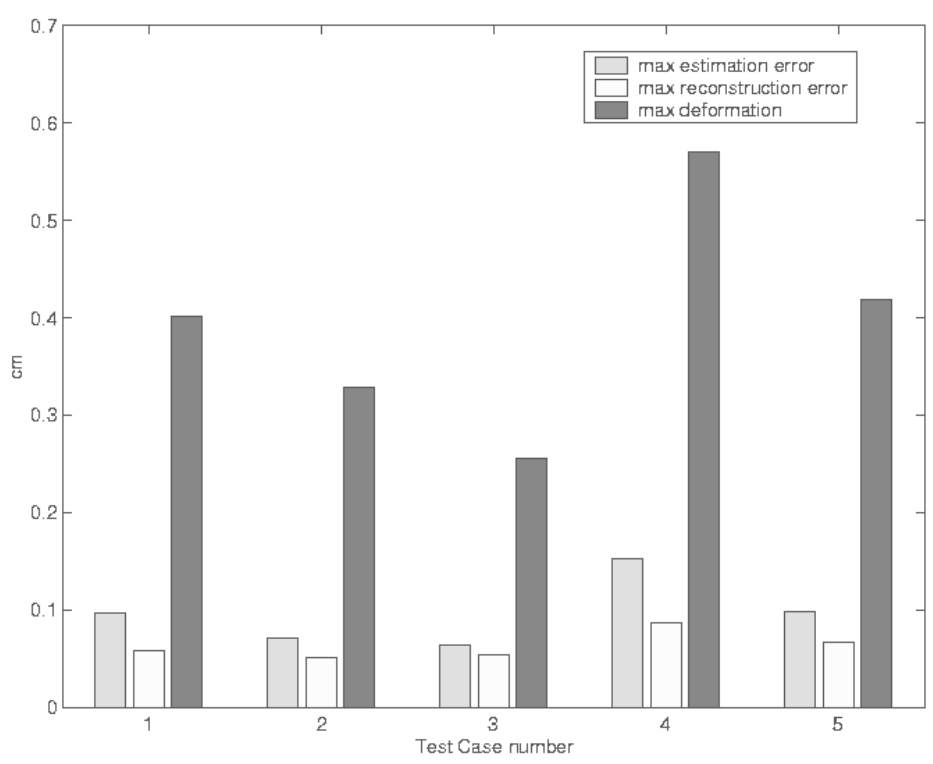

Fig. 3. The computed maximum estimation error, reconstruction error, and deformation of the prostate for 5 different simulations of TRUS probe insertion.

deformation encountered in this simulation. However, the reconstruction error accounted for more than $57 \%$ of the estimation error for this case. The availability of more training samples obtained from more biomechanical simulations will reduce this error, at the expense of increased computational burden.

\section{Summary and Future Work}

We presented an approach that combines biomechanical and statistical modeling for estimation of the shape of the prostate deformed during TRUS probe insertion. Our approach makes use of a patient specific biomechanical model constructed from a segmented volumetric scan of the patient's prostate. Since it is virtually not possible to perform biomechanical simulations for every possible value of probe displacement and entry angles, only a small number of biomechanical simulations are used to extract the modes of deformation of the prostate. The coefficients of those modes were then related to the parameters of the biomechanical simulation, namely, the insertion angles and insertion depth of the TRUS probe. This enabled the parameterization of the deformed prostate shape in terms of the biomechanical simulation parameters, and therefore provided a means for combined estimation of a set of deformed prostate shapes given sparse $2 \mathrm{D}$ ultrasound images.

The framework of [3] can be used to extend the approach presented here to a deformable model for the prostate that includes the modes of deformation as well as modes of shape. Such model can be constructed from several subjects instead of using a patient specific biomechanical model. Another possible extension to 
the work presented here is the treatment of material parameters as another simulation variable that is related to the modes of deformation, and seeking an estimate of those values as a part of the optimization step.

\section{Acknowledgement}

The work reported in this paper was supported in part by the National Science Foundation under Engineering Research Center grant EEC9731478, and by the National Institutes of Health grant R01NS42645.

\section{References}

1. Bharatha, A., Hirose, M., Hata, N., Warfield, S., Ferrant, M., Zou, K.H., SuarezSantana, E., Ruiz-Alzola, J., D’Amico, A., Cormack, R.A., Kikinis, R., Jolesz, F.A., Temapny, C.M., Evaluation of Three-Dimensional Finite Element-Based Deformable Registration of Pre- and Intraoperative Prostate Imaging. Medical Physics 28(12) December (2001) 2551-2560

2. Booth, J.T., Zavgorodni, S.F., Set-up Error and Organ Motion Uncertainty: a Review. Australas Phys. Eng. Sci. Med., Jun; 22(2) (1999) 29-47

3. Davatzikos, C., Shen, D., Mohamed, A., Kyriacou, S.K., A Framework for Predictive Modeling of Anatomical Deformations. IEEE Transactions on Medical Imaging 20(8) August (2001) 836-843

4. Fleute, M., Lavallée, S., Nonrigid 3-D/2-D Registration of images Using Statistical Models. Lecture Notes in Computer Science, Vol. 1679. Medical Image Computing and Computer Assisted Intervention 1999 Springer-Verlag, Berlin Heidelberg New York (1999) 138-147

5. Ferrant, M., Warfield, S.K., Guttmann, C.R., Mulkern, R.V., Jolesz, F.A., Kikinis, R., 3D Image Matching Using a Finite Element Based Elastic Deformation Model. Lecture Notes in Computer Science, Vol. 1679. Medical Image Computing and Computer Assisted Intervention 1999 Springer-Verlag, Berlin Heidelberg New York (1999) 202-209

6. Sullivan, J.M., Charron, G., Paulsen, K.D., A Three-Dimensional Mesh Generator for Arbitrary Multiple Material Domains. Finite Elements in Analysis and Design 25 (1997) 219-241

7. Abaqus version 6.1. Hibbitt, Karlsson, and Sorensen, Inc., USA, 2000.

8. Weaver, J.B., Van Houten, E.E., Miga, M.I., Kennedy, F.E., Paulsen, K.D., Magenetic Resonance Elastography Using 3D Gradient Echo Measurements of SteadyState Motion. Medical Physics 28(8) August (2001) 1620-1628

9. Jolliffe, I.T., Principal Component Analysis. Springer-Verlag, Berlin Heidelberg New York (1986)

10. Farin, G., Curves and Surfaces for Computer Aided Geometric Design. Academic Press Limited, London, UK (1997)

11. Nelder, J.A., and Mead, R., A Simplex Method for Function Minimization. Computer J. 7 (1965) 308-313

12. Mohamed, A., Kyriacou, S.K., Davatzikos, C., A Statistical Approach for Estimating Brain Tumor Induced Deformation. Mathematical Models in Biomedical Image Analysis (2001) 52-59 